\title{
L'ASSM rejette la réglementation proposée de l'assistance organisée au suicide
}

Académie Suisse des Sciences Médicales (ASSM)
1 Rietjens JA et al. A comparison of attitudes towards end-of-life decisions: survey among the Dutch general public and physicians. Soc Sci Med. 2005;61(8):1723-32.

Correspondance:

ASSM

Petersplatz 13

CH-4051 Bâle

mail@samw.ch
Le Conseil Fédéral a décidé de réglementer l'assistance organisée au suicide; fin octobre 2009, il a mis en consultation deux variantes différentes. La variante 1, privilégiée par le Conseil Fédéral, prévoit l'introduction de critères de diligence rigoureux. Dans l'art. 115 al. 2 du Code pénal, sept conditions à remplir par un accompagnant au suicide actif au sein d'une association sont énoncées:

- la décision de se suicider est prise librement, mûrement réfléchie et persistante;

- un médecin indépendant de l'organisation atteste que le suicidant est capable de discernement;

- un second médecin indépendant de l'organisation atteste que le suicidant souffre d'une maladie incurable avec une issue fatale imminente;

- des alternatives de traitement ont été discutées avec le suicidant et lui ont été proposées;

- le moyen employé est soumis à prescription médicale;

- l'accompagnant ne poursuit pas de but lucratif;

- l'organisation et l'accompagnant constituent conjointement une documentation complète.

La variante 2 du projet prévoit une interdiction générale de l'assistance organisée au suicide.

L'Académie Suisse des Sciences Médicales (ASSM) a minutieusement examiné le nouveau projet de réglementation et soumis la prise de position suivante au Conseil Fédéral.

\section{La réglementation prévue de l'assistance organisée au suicide ne résout pas les problèmes existants}

Depuis des décennies, les questions relatives à l'assistance et à l'accompagnement au décès représentent un thème central pour l'Académie Suisse des Sciences Médicales (ASSM). Dans ses directives médico-éthiques, elle a défini des normes pour l'activité médicale dans ce domaine sensible. L'ASSM a toujours défendu sa position, selon laquelle une législation sur l'euthanasie indirecte active ou passive est superflue et considère que ses directives constituent un outil adéquat. En revanche, l'ASSM plaide depuis longtemps en faveur d'un contrôle des activités des organisations d'assistance au suicide. Dans un courrier adressé au Conseil Fédéral le 7 juin 2006, elle écrivait à ce sujet:

«Nous considérons que la protection d'une personne suicidaire est absolument décisive ... Nous ne pouvons nous satisfaire de l'insuffisance des critères de diligence et du manque de surveillance dans un domaine où il est question de vie et de mort ... Nous sommes clairement de l'avis que la Confédération a un devoir de surveillance dans le domaine des organisations d'assistance au suicide.»

En principe, l'ASSM salue les efforts de la Confédération pour réglementer l'assistance organisée au suicide. Elle soutient notamment son intention de ne réglementer qu'une partie de la pratique de l'assistance au suicide, à savoir l'assistance organisée. Elle pense toutefois que ni la limitation proposée à l'assistance organisée au suicide (variante 1), ni son interdiction (variante 2) - par une réglementation dans le Code pénal resp. dans le Code pénal militaire - ne représente une solution adéquate aux problèmes actuels. L'ASSM rejette la réglementation proposée pour les raisons suivantes:

- L'assistance au suicide sera institutionnalisée en tant qu'activité médicale.

- Les institutions médicales seront soumises à une pression accrue les incitant à accepter que les organisations d'assistance au suicide interviennent dans leurs locaux.

- La limitation à la «fin de vie imminente» est inadéquate.

- La réglementation proposée peut être facilement contournée.

\section{L'assistance au suicide sera institutionnalisée} en tant qu'activité médicale

La nouvelle réglementation, telle qu'elle est proposée, impliquerait de plus en plus les médecins dans l'assistance au suicide. En effet, dans la variante 1, trois tâches centrales incomberaient aux médecins (la constatation de la fin de vie, la vérification de la capacité de discernement et la prescription de pentobarbital de sodium [NAP]) et les soumettraient à une pression accrue pour qu'ils pratiquent l'assistance au suicide resp. se chargent de tâches liées à un suicide planifié. Dans les variantes 1 et 2 , la limitation à l'assistance organisée au suicide, telle qu'elle est prévue dans la réglementation, pourrait inciter les malades chroniques suicidaires, qui ne sont pas en fin de vie, à faire de plus en plus appel aux médecins. Etonnamment ces conséquences sur le rôle des médecins ne sont pas évoquées dans le rapport explicatif.

Dans les directives de l'ASSM «Prise en charge des patientes et patients en fin de vie» l'assistance médicale au suicide est tolérée en tant que décision morale personnelle du médecin, dans certains cas particuliers et dans les limites d'un cadre déterminé. Cependant, l'ASSM a maintes fois précisé que l'établissement croissant de l'assistance au suicide relève de la responsabilité de la société et que celle-ci ne peut être déléguée au corps médical. L'attitude du médecin individuel dans la marge de manœuvre définie par le droit dépend aujourd'hui de sa propre conscience. L'expérience montre que l'assistance au suicide est mieux acceptée dans la population qu'au sein du corps médical [1] et plus les médecins concernés ont de l'expérience avec la prise en charge de patients en fin de vie, moins ils adhèrent à l'assistance au suicide [2]. 
Les expériences acquises au niveau international révèlent, elles aussi, qu'à cet égard le potentiel de conflit est en étroite relation avec la perception individuelle de l'activité médicale. L'accompagnement d'un être souffrant en fin de vie et son soutien dans les moments difficiles représentent des missions importantes pour les médecins; en revanche, ils ne considèrent pas comme étant de leur devoir d'apporter une aide au suicide à des personnes souffrant de maux divers (comme la solitude, l'isolement, le handicap ou une maladie psychique) et qui ne sont pas en fin de vie. Actuellement, $30 \%$ des cas d'assistance organisée au suicide ne concernent pas des personnes en fin de vie [3]. C'est pourquoi, il est probable qu'avec la nouvelle réglementation (variante 1 et 2 ) la pression exercée sur le médecin individuel à pratiquer l'assistance au suicide risque d'être de plus en plus forte.

Avec la variante 1 de la réglementation, le corps médical est encore plus impliqué dans l'assistance au suicide, car seuls les médecins sont responsables de la constatation de la capacité de discernement. Selon l'ASSM, ce n'est qu'en présence de doutes concernant des questions médicales (par ex. lors de la vérification de la capacité de discernement chez des patients atteints d'un début de démence ou de troubles psychiques) qu'un deuxième avis doit être établi par un médecin.

Les institutions médicales seront soumises à une pression accrue les incitant à accepter que les organisations d'assistance au suicide interviennent dans leurs locaux

Nombreux sont ceux qui passent la dernière phase de leur vie à l'hôpital ou dans un lieu de vie médicalisé (institution de soins). En limitant l'assistance organisée au suicide aux patients en fin de vie - comme le prévoit la variante 1 - les hôpitaux et institutions de soins seraient soumis à une pression de plus en plus forte les incitant à accepter que les organisations d'assistance au suicide interviennent dans leurs locaux, alors que l'assistance au suicide est en contradiction avec le rôle initial de ces institutions. Si pour cette raison, la plupart des hôpitaux persistaient à interdire leur accès aux organisations d'assistance au suicide, cela équivaudrait en fait à l'interdiction de l'assistance organisée au suicide (variante 2), interdiction que le Conseil fédéral n'encourage pas.

\section{La limitation de la réglementation à la «fin de vie} imminente» est inadéquate

L'ASSM a introduit dans ses directives le critère de «fin de vie», dans le but d'aider les médecins en charge de patients mourants, dans les situations limites où ils sont confrontés à une demande de suicide. Toutefois, la «fin de vie» n'est pas un critère général adapté à la réglementation de l'assistance organisée au suicide. D'une part, les difficultés liées au pronostic de la fin de vie sont connues et, d'autre part, une telle limitation est contraire à l'opinion et aux attentes d'une grande partie de la population.

\section{La réglementation proposée peut être facilement contournée}

Finalement, les problèmes actuels ne peuvent pas être résolus avec les variantes proposées, car les limitations peuvent être facilement contournées: Les conditions énoncées dans la législation s'adressent à des organisations et non pas à des individus ou des médecins. Ainsi, les personnes suicidaires qui ne remplissent pas les conditions nécessaires peuvent être adressées par l'organisation à des médecins qui eux ne sont pas soumis à la nouvelle réglementation.

\section{Propositions de réglementation à l'aide d'une loi spécifique}

Dans son rapport intitulé «Assistance au décès et médecine palliative - la Confédération doit-elle légiférer?» du 24 avril 2006, le Département Fédéral de la Justice et de la Police avait envisagé diverses possibilités pour éviter les malentendus dans le domaine de l'assistance au suicide. Outre la révision de l'art. 115 du Code Pénal, le DFJP avait également proposé une réglementation au moyen d'une loi de surveillance. Sur la base des conclusions de ce rapport, le Conseil Fédéral avait renoncé à faire une proposition de réglementation en mai 2006.

Depuis lors, aussi bien des initiatives parlementaires (par ex. les motions $\mathrm{N}^{\circ} 073163$ et 07.3626) que la Commission Nationale d'Ethique (CNE) et l'ASSM exigent du Conseil Fédéral qu'il élabore une base juridique pour le contrôle des organisations d'assistance au suicide. Toutefois, la possibilité d'une réglementation de surveillance n'est abordée que brièvement dans le chapitre 4.3 du rapport concernant le projet de révision du Code Pénal en argumentant qu'avec une telle réglementation une certaine bureaucratisation de l'assistance organisée au suicide serait inévitable. Selon l'ASSM, une législation de surveillance pour l'assistance organisée au suicide représenterait une solution adéquate, car d'une part, les organisations de suicide assisté seraient soumises à autorisation et, d'autre part, il serait possible de contrôler si les critères de diligence définis sont respectés.

Les points suivants doivent être réglés dans la loi de surveillance:

- Les devoirs de diligence pour les assistants au suicide (en particulier le choix minutieux des personnes, une formation adéquate et la supervision).

- Les devoirs de diligence pour les médecins qui travaillent avec les organisations d'assistance au suicide, en particulier pour les médecins qui établissent les prescriptions.

- La transparence de la gestion et des statistiques.

\section{Conclusion}

Sur la base des déclarations ci-dessus, l'ASSM rejette les propositions de règlementation (variantes 1 et 2 ) et propose une règlementation au moyen d'une loi de surveillance pour les organisations d'assistance au suicide. Elle recommande par ailleurs à la Confédération de renforcer la prévention du suicide et de continuer à promouvoir les soins palliatifs. 